\title{
Dental Sleep Medicine in the Dental Curriculum: What Should Be the Dot on the Horizon?
}

\author{
Frank Lobbezoo, DDS, $\mathrm{PhD}^{1}$; Ghizlane Aarab, DDS, $\mathrm{PhD}^{1}$ \\ ${ }^{1}$ Department of Orofacial Pain and Dysfunction, Academic Centre for Dentistry Amsterdam (ACTA), University of Amsterdam and Vrije \\ Universiteit Amsterdam, Amsterdam, The Netherlands
}

Dental sleep medicine is a relatively new offshoot of dentistry. The discipline is characterized by growth over the past two decades with the number of publications containing the term 'dental sleep medicine' in Web of Science (All Databases; Topic search) growing from 50 in 1999 to $>300$ in 2020. This impressive growth is likely related to the importance of dental sleep medicine for general health issues. Many of the so-called dental sleep disorders, i.e., sleep disorders that have important diagnostic and/or management links to the dental domain, may have a large impact on general health. These conditions include snoring and obstructive sleep apnea as well as sleep-related orofacial pain, xerostomia, hypersalivation, gastroesophageal reflux disease, and bruxism) $[1,2]$.

Thus, as is the case for the dentist's involvement in managing periodontal problems [3], dentists not only contribute to maintaining and improving the oral health of their patients, but also to managing their patients' general health. As listed above, several sleep disorders have been recognized as being in the dental domain, but it remains to be determined whether or not this set of disorders is exhaustive. Currently, an international project is aiming to identify all sleep-related problems in which a dentist could potentially play a role in the recognition, prevention, and/or management. Pending the outcome of this project, research has already indicated that many, if not all dental sleep disorders are linked to each other as multi-morbid conditions, something to take into account when dealing with dental sleep disorders in practice.

A key question now is: what should the dot on the horizon be regarding the acquisition of competencies in dental sleep medicine by general dentists and other professionals in oral health care? Given the high and increasing prevalence of (dental) sleep disorders and their enormous impact on individual patients and society, we are of the opinion that all general dentists, supported by other professionals who are active in dentistry (including dental hygienists and dental nurses), should be competent in recognizing, preventing, and managing dental sleep disorders. Clearly, if only dentists specializing in dental sleep medicine are responsible for aspects of dental sleep disorders, only a very small proportion of patients can be helped. Hence, specialists in dental sleep medicine should focus on more complex, multi-morbid cases in whose diagnosis and treatment several medical specialists are involved working in interdisciplinary teams.

It should be stressed that the above-sketched dot on the horizon has not yet been reached. On the contrary, there is still a long way to go. In a recent commentary published in the European Journal of Dental Education [4], the authors highlighted the relevance of dental sleep medicine in the general dental education and proposed potential educational content for integration in the dental curriculum. A main focus of the commentary was on obstructive sleep apnea, this disorder being highly prevalent and potentially lethal. However, we would like to add that since dental sleep disorders are so intensely interrelated with one another, sufficient attention should be given to other dental sleep disorders as well.

It is now up to policy makers and designers of dental and oral health-related curricula at all levels (i.e., undergraduate, graduate, and postgraduate) to formulate relevant competencies, build courses, and design exams that will enable us to train oral health care professionals who are able to recognize and manage dental sleep disorders. During that process, it is inevitable that in some countries the dot on the horizon will be reached faster than in other countries. In The Netherlands, for example, the following competency has recently been included in the undergraduate dental curriculum nationwide: "... treats obstructive sleep apnea syndrome or refers the patient for it." Consequently, relevant courses are currently being built and appropriate exams are being designed to ascertain that graduating general dentists will indeed have the competency to deal with this topic independently. Of course, to reach expert levels, several years of experience and additional training are needed, as is the case for all competencies within dentistry.

In conclusion, it is our opinion that recognizing, preventing, and managing dental sleep disorders should be part of the competencies of all general dentists, supported by other oral health care professionals, including dental hygienists and dental nurses. This would allow most patients who suffer from a dental sleep disorder, especially a non-complex disorder, to receive the necessary professional help while in the first line of care. More complex cases would then be referred to dentists 
specializing in dental sleep medicine working in dedicated interdisciplinary teams. This vision will require appropriate training for all oral health care providers. It is up to policy makers and designers of dental curricula to make this happen.

\section{CITATION}

Lobbezoo F, Aarab G. Dental sleep medicine in the dental curriculum: What should be the dot on the horizon?. J Dent Sleep Med. 2021;8(2).

\section{REFERENCES}

1. Lavigne GJ, Goulet JP, Zuconni M, Morisson F, Lobbezoo F. Sleep disorders and the dental patient: A review of diagnosis, pathophysiology and management. Oral Surg Oral Med Oral Pathol Oral Radiol Endod. 1999;88:257-272.

2. Lobbezoo F, Aarab G, Wetselaar P, Hoekema A, Lange J de, Vries N de. A new definition of Dental Sleep Medicine. J Oral Rehabil. 2016;43:786-790.

3. Teeuw WJ, Gerdes VE, Loos BG. Effect of periodontal treatment on glycemic control of diabetic patients: a systematic review and meta-analysis. Diabetes Care. 2010;33:421-427.

4. Herrero Babiloni A, Beetz G, Dal Fabbro C, Martel MO, Huynh N, Masse JF, Sessle B, Lavigne GJ. Dental sleep medicine: Time to incorporate sleep apnoea education in the dental curriculum. Eur J Dent Educ. 2020;24:605-610.

\section{SUBMISSION AND CORRESPONDENCE INFORMATION}

\section{Submitted for publication January 25, 2021 \\ Accepted for publication February 7, 2021}

Address correspondence to: Prof. Frank Lobbezoo, Department of Orofacial Pain and Dysfunction, Academic Centre for Dentistry Amsterdam (ACTA), Gustav Mahlerlaan 3004, 1081 LA Amsterdam, the Netherlands, f.lobbezoo@acta.nl

\section{DISCLOSURE STATEMENT}

This paper was previously published in Sleep and Breathing (citation: Lobbezoo F, Aarab G. Dental sleep medicine in the dental curriculum: what should be the dot on the horizon? Sleep Breath. 2020 Jun 24. doi: $10.1007 / \mathrm{s} 11325-020-02133-\mathrm{x})$ and is republished with permission from Springer Nature (Copyright Clearance Center License Number 4993640054774).

Frank Lobbezoo is a member of the Academic Advisory Boards for GrindCare and Oral Function of Sunstar Suisse S.A. and receives research grants from Sunstar Suisse S.A., SomnoMed, Vivisol-Netherlands BV, Health Holland, and Airway Management. Ghizlane Aarab is a member of the Academic Advisory Board for Oral Function of Sunstar Suisse S.A. and receives research grants from Sunstar Suisse S.A., SomnoMed, VivisolNetherlands BV, and Health Holland. 